LPTHE-ORSAY Preprint 94-84

September 1994

\title{
Fermion masses and mixing from an extra gauge symmetry.
}

\section{E. Papageorgiu [}

Laboratoire de Physique Théorique et Hautes Energies Université de Paris XI, Bâtiment 211, 91405 Orsay, France.

Looking for simplicity could mean looking for a symmetry. For Feza Gürsey this was more than a hypothesis or just an attitude. It was a rewardfull commitment he shared with other physicists. Following the same attitude one may hope to find an underlying symmetry principle for the long list of mass parameters which characterize the elementary particle spectrum and enter in the Standard Model (SM) as free parameters. To start with, one can look for regularities in terms of some observable. We know for example that the observed mass and mixing hierarchies of the fermion spectrum can be described in terms of the Wolfenstein parameter $\lambda \simeq 0.22$ which, to a good approximation, gives the Cabbibo mixing $\left|V_{u s}\right|$. Taking into account the experimental uncertainties that can be as large as a factor of two one finds the following mass patterns for the up and down quarks:

$$
\begin{aligned}
& m_{u}: m_{c}: m_{t} \sim\left(\frac{\lambda^{8}}{\chi^{3}}: \frac{\lambda^{4}}{\chi^{3}}: 1\right) \times m_{t} \\
& m_{d}: m_{s}: m_{b} \sim\left(\frac{\lambda^{4}}{\chi}: \frac{\lambda^{2}}{\chi}: 1\right) \times m_{b} .
\end{aligned}
$$

\footnotetext{
${ }^{1}$ Talk given at the Gürsey Memorial Conference on Strings and Symmetries I, June 6-10, 1994, Istanbul, Turkey.
} 
The parameter $\chi \sim 0.7$ gives the radiative corrections (from the heavy top quark) to the mass ratios when they run from the unification scale $M_{G}=$ $10^{16} \mathrm{GeV}$ down to the electroweak scale according to the renormalisation group equations of the minimal supersymmetric standard model (MSSM). This effect is particularly felt in the up-quark sector. The regularity of the spectra in terms of $\lambda$ becomes more transparent at the very high scales, where one also notices that ratios of up quark masses and down quark masses are related through a $\lambda^{2} \leftrightarrow \lambda$ transformation. Compared to the almost equal spacing between neighbouring quark mass levels the charged lepton masses are perceived as an anomaly

$$
m_{e}: m_{\mu}: m_{\tau} \sim\left(\lambda^{5}: \lambda: 1\right) \times m_{\tau}
$$

even at the unification scale where apart from the unification of gauge couplings one has also partial unification of Yukawa couplings and the mass relations:

$$
m_{\tau} \simeq m_{b} \quad \text { and } \quad m_{e} \cdot m_{\mu} \simeq m_{d} \cdot m_{s}
$$

Given the fact that at low energies one has only 13 observables (six quark and three lepton masses, the three mixing angles and the $\mathrm{CP}$ violating phase of the CKM matrix) one cannot fix the entries of the quark and lepton mass matrices $M_{u}, M_{d}$ and $M_{e}$ at $M_{G}$, even by assuming that the latter are symmetric. This has led to different Ansätze [1,2] in which some of the entries are zero while the others are given in powers of $\lambda$. In the quark sector the maximum number of zeros that one can have is five (counting together those in $M_{u}$ and $M_{d}$, but without counting symmetric entries twice) and there are five different pairs of $M_{u}-M_{d}$ textures at $M_{G}$ that lead to masses and mixings which are compatible with the present-day experimental values [2]. Some extra consideration is thus needed to single out a unique solution. In fact, the zeros in the mass matrices can be thought off as "relics" of a new symmetry which is not "family-blind", while the small non-zero entries could well be correction terms generated after symmetry breaking. I will discuss this "old" idea in the light of a new way of obtaining also the successful $\sin ^{2} \theta_{W}=3 / 8$ result of the canonical gauge coupling unification which consists in extending the gauge group of the standard model by a horizontal $U(1)_{X}$ factor whose anomalies can be cancelled by a Green-Schwarz mechanism [3].

Let us assume the existence of a family-dependent $U(1)_{X}$ gauge symmetry at $M_{\text {Planck, }}$, with respect to which the quarks and leptons carry charges $\alpha_{i}$ and 
$a_{i}$ respectively, where $i=1,2,3$ is the generation index. We first consider the up quark mass matrix. Given the predominant role played by the top quark it is not unnatural to start with a rank-one matrix and make a choice for the charges such that only the $(3,3)$ renormalizable coupling $t^{c} t h_{1}$ is allowed. This fixes the charge of the light Higgs $h_{1}$ to $-2 \alpha\left(\alpha \equiv \alpha_{3}\right)$. We expect the other entries to be generated by higher-dimension operators which may occur at the string compactification level and contain combinations of scalar fields some of which acquire vacuum expectation values (vev's) leading to spontaneous symmetry breaking and large masses for the non-observable part of the spectrum. For simplicity let us assume a pair of singlet fields $\sigma_{ \pm}$developping equal (vev's) along a "D-flat" direction and carrying opposite charges \pm 1 . They can give rise to higher-order couplings $q_{i}^{c} h_{1}\left(\frac{\langle\sigma\rangle}{M}\right)^{\left|2 \alpha-\alpha_{i}-\alpha_{j}\right|} q_{j}$. Notice that when the exponent is positive (negative) only the field $\sigma_{+}\left(\sigma_{-}\right)$can contribute. The new scale $\mathcal{E}=\frac{\langle\sigma\rangle}{M}$ which enters in the quark mass matrix is the ratio of the symmetry breaking scale to the scale that governs these higherdimension operators, and could be the string unification scale $M_{S} \simeq 10^{18}$ $\mathrm{GeV}$ or $M_{\text {Planck. }}$. If $\mathcal{E}$ is a small number one finds two universal hierarchy patterns in the generated texture:

$$
M_{x} \sim\left(\begin{array}{ccc}
\mathcal{E}^{2\left|x_{1}\right|} & \mathcal{E}^{\left|x_{1}+x_{2}\right|} & \mathcal{E}^{\left|x_{1}\right|} \\
\mathcal{E}^{\left|x_{1}+x_{2}\right|} & \mathcal{E}^{2\left|x_{2}\right|} & \mathcal{E}^{\left|x_{2}\right|} \\
\mathcal{E}^{\left|x_{1}\right|} & \mathcal{E}^{\left|x_{2}\right|} & 1
\end{array}\right) \quad\left|x_{1,2}\right|=\left|\alpha-\alpha_{1,2}\right|
$$

namely $m_{11} \sim m_{13}^{2}$ and $m_{22} \sim m_{23}^{2}$. The choice $\left|x_{2}\right|=1$ and $\left|x_{1}\right|=4$ or $\left|x_{1}\right|=2$ leads to:

$$
M_{u}^{F} \sim\left(\begin{array}{ccc}
\mathcal{E}^{8} & \mathcal{E}^{3} & \mathcal{E}^{4} \\
\mathcal{E}^{3} & \mathcal{E}^{2} & \mathcal{E} \\
\mathcal{E}^{4} & \mathcal{E} & 1
\end{array}\right) \quad \text { or } \quad M_{u}^{G} \sim\left(\begin{array}{ccc}
\mathcal{E}^{4} & \mathcal{E}^{3} & \mathcal{E}^{2} \\
\mathcal{E}^{3} & \mathcal{E}^{2} & \mathcal{E} \\
\mathcal{E}^{2} & \mathcal{E} & 1
\end{array}\right)
$$

If $\mathcal{E}$ is of order $\lambda^{2}$ the two textures above correspond to the phenomenologically acceptable Ansätze à la Fritzsch fl or à la Giudice for the up quark mass matrix. The generation of other acceptable textures having a zero in the $(2,2)$ or the $(2,3)$ entry (but not in both entries simultaneously) necessitates a more complicated mechanism involving extra singlets and mixing with heavy Higgses.

\footnotetext{
${ }^{2}$ The original Ansatz had a zero in the $(2,2)$ entry.
} 
The next task is, given the up quark matrices of eq.(6), to construct an acceptable down quark matrix. The assumption of symmetric mass matrices and the $S U(2)_{L}$ symmetry require the equality between the charges of the up and down quarks. Assuming again that only the $(3,3)$ renormalizable coupling is allowed leads to the other light Higgs $h_{2}$ carrying the same charge as $h_{1}$. This means that this $U(1)_{X}$ is anomalous and needs a cancellation mechanism which will be discussed at the end of the talk. Notice that the choice of a particular texture for $M_{u}$ has already fixed the texture of $M_{d}$ in terms of some new scale $\mathcal{E}^{\prime}$ which has to be of order $\lambda$ to give the correct mass spectrum of eq.(2). The origin of this difference in scale $\mathcal{E}^{\prime} \sim \mathcal{E}^{1 / 2}$ is yet unknown.

Thus if one insists in generating the up and down quark mass matrices through the same (simple) mechanism that led to eq.(5) one can only generate textures à la Fritzsch containing four zeros in total. In order to generate the five-zero textures of ref.[2] some sort of extension is needed. In many compactification schemes there are among other things additional pairs of Higgs fields which acquire masses at the scale of symmetry breaking. One can thus envisage the possibility of mixing between the light Higgses $h_{1,2}$ with heavy Higgses $H_{1,2}$ whose charge we denote by $-2 \beta$. Then one can also have couplings $q_{i}^{c} H\left(\frac{<\sigma>}{M}\right)^{\left|2 \beta-\alpha_{i}-\alpha_{j}\right|} q_{j}$ which give rise to the following texture:

$$
M_{z} \sim\left(\begin{array}{ccc}
\mathcal{E}^{2\left|z_{1}\right|} & \mathcal{E}^{\left|z_{1}+z_{2}\right|} & \mathcal{E}^{\left|z_{1}+z\right|} \\
\mathcal{E}^{\left|z_{1}+z_{2}\right|} & \mathcal{E}^{2\left|z_{2}\right|} & \mathcal{E}^{\left|z_{2}+z\right|} \\
\mathcal{E}^{\left|z_{1}+z\right|} & \mathcal{E}^{\left|z_{2}+z\right|} & 1+\mathcal{E}^{2|z|}
\end{array}\right),
$$

whith $\left|z_{i}\right|=\left|\beta-\alpha_{i}\right|$, and $|z|=|\beta-\alpha|$. When the difference between the light- and heavy-Higgs charges is larger than between the charges of the heavy Higgs and the quarks this automatically gives suppressed $(1,3)$ and $(2,3)$ mass entries. Taken together, the textures of eqs. $(5,7)$ can generate any acceptable up or down quark texture. On the other hand the generation of an acceptable set of up and down quark matrices with five zeros is again too constrained, while the Fritzsch Ansatz can be obtained also from the conditions $\left|x_{2}\right|=1,\left|z_{1}+z_{2}\right|=3$ and $\left|x_{1}\right|,\left|z_{1,2}\right|,|z| \gg 0[4]$.

What about the lepton sector? Assuming simply the gauge symmetries of the SM the $U(1)_{X}$ charges $a_{i}$ of the leptons are not related to those of the quarks. Allowing however the coupling $\tau^{c} \tau h_{2}$ leads to $a_{3}=\alpha$ and to $m_{\tau}=m_{b}$ unification. Another constraint comes from the second mass relation of eq.(4) 
which means that we should be looking for textures for which det $M_{e}=$ det $M_{d}$ and therefore for combination of charges that satisfy: $a_{1}+a_{2}=$ $\alpha_{1}+\alpha_{2}$. Since the early days of grand unification it is known that in order to obtain also for the first two generations acceptable mass relations between the charged leptons and down quarks the $(2,2)$ entry of $M_{d}$ should be multiplied by a factor of minus three, the other entries of $M_{e}$ and $M_{d}$ been equal. In this way, though there can be no explanation of the factor minus three in this approach, in terms of the scale $\mathcal{E}^{\prime}$ and its various powers, $M_{e}$ becomes identical with $M_{d}$. The alternative is a texture:

$$
M_{e} \sim\left(\begin{array}{ccc}
0 & \lambda^{3} & 0 \\
\lambda^{3} & \lambda & 0 \\
0 & 0 & 1
\end{array}\right)
$$

which can be generated from the texture $M_{z}$ in eq.(7) from the choices $\left|z_{2}\right|=$ $1 / 2$ and $\left|z_{1}\right|=5 / 2$ or $7 / 2$ when $|z| \gg\left|z_{1,2}\right|$.

We turn now to the neutrino sector. Again as a consequence of the $S U(2)_{L}$ symmetry and our symmetric Ansatz the lefthanded and righthanded neutrinos, $\nu_{i}$ and $N_{i}$, become charged under the $U(1)_{X}$ with the same charges $a_{i}$ as the charged leptons. Obviously the presence of the $N_{i}$ 's implies a larger symmetry than what has been assumed so far, but also the assumption of symmetric mass matrices can find its justification only in the context of a left-right symmetric theory. Starting from $M_{e}=M_{d}$ and assuming that the same mechanism which generated masses for the quarks and the leptons generates also Dirac masses for the neutrinos we are automatically led to another well known GUT equality: $M_{\nu}^{D}=M_{u}$. In the same way starting from eq.(8) one obtains $M_{\nu}^{D}=M_{e}$. On the other hand, Majorana mass terms $M_{R}^{i j}$ need not be generated in the same way. In compactified string models, due to the absence of large Higgs representations, righthanded neutrinos donot get tree-level masses, so all entries in $M_{R}$ are due to nonrenormalizable operators, and nothing is a priori known concerning the particular texture of $M_{R}$ or the existence of a possible hierarchy in this sector. The only constraints come from the requirement that the seesaw-suppressed masses of the ordinary neutrinos should be below the experimental upper limits. For this, $M_{R}$ has to be a nonsingular matrix and its scale should be well above the electroweak scale. Therefore in addition to the operators that generated the textures of eqs. $(5,7)$ one will need at least an extra piece to set the Majorana mass scale. 
Common examples are operators containing the heavy Higgses which have been used for generating the texture in eq.(7), namely $N_{i}^{c} H H N_{j}$, whose scale is of $\mathcal{O}\left(M_{G}^{2} / M_{S}\right)$ multiplied for some orbifold suppression factor. In this case the condition $\alpha_{i}+\alpha_{j}=4 \beta$ leads to $M_{R}^{i j} \neq 0$. One can always fix the charges of the light and heavy Higgses relative to each other such that there is at least one entry which is different from zero. On the other hand, the charges $\alpha_{1}, \alpha_{2}$ (and $\left.a_{1}, a_{2}\right)$ are fixed by the conditions which led to eqs. $(6,8)$ in terms of the Higgs charges. Therefore there is no freedom in generating more entries through the same operator. There are two alternatives paths leading to the generation of a nonsingular $M_{R}$ : Either some of the Majorana entries are generated perturbatively in a similar way as the entries in the other mass matrices (this implies a hierarchy of righthanded neutrino scales) or one can assume a different set of singlets in the higher-dimension operators which give rise to the texture $M_{z}$ in eq.(7). This freedom allows the generation of

$M_{R}$ textures containing four zeros [4] and leading to interesting predictions for neutrino oscillation experiments [5].

Let us finally comment on the cancellation of the mixed anomalies of a $U^{\prime}(1)$ with $S U(3), S U(2)$ and $U(1)_{Y}$ via a Green-Schwarz (GS) term in $4 \mathrm{D}$ string theories. It was pointed out that the existence of an axion with universal couplings to all gauge groups together with the assumption that the gauge couplings, up to normalisation factors $k_{i}$, meet at some scale, can lead to cancellation of the anomalies by an appropriate shift of the axion field but also to gauge coupling unification if the anomaly coefficients are in the ratio $A_{3}: A_{2}: A_{1}=k_{3}: k_{2}: k_{1}=1: 1: 5 / 3$ [6]. It is remarkable that this seems to be the case also for the $U(1)_{X}$ that gave an acceptable mass spectrum [3].

\section{References}

[1] H. Fritzsch, Phys. Lett. B 70(1977)436; Phys. Lett. B 73(1978)317; Nucl. Phys. B 155(1979)189; J. Harvey, D. Reiss and P. Ramond, Phys. Lett. B 92(1980)309; Nucl. Phys. B 199(1982)223; S. Dimopoulos, L.J. Hall and S. Raby, Phys. Rev. Lett. 68(1992)1984; Phys. Rev. D 45(1992)4192; G. F. Giudice, Mod. Phys. Lett. A7(1992)2429. S. Dimopoulos, L.J. Hall and S. Raby, Phys. Rev. D 47(1993)3697; L.J. Hall, UCB-PTH-92-22. 
[2] P. Ramond, R.G. Roberts and G.G. Ross, Nucl. Phys. B 406(1993)19.

[3] L.E. Ibáñez and G.G. Ross, Phys. Lett. B 332(1994)100.

[4] E. Papageorgiu, "Yukawa textures from extra $U(1)$ symmetry?", LPTHE-ORSAY Preprint 94-40, Zeit. Phys. C 64(1994)509.

[5] E. Papageorgiu, "Neutrino properties from maximally predictive GUT models and the structure of the heavy Majorana sector", LPTHEORSAY Preprint 93-45, Zeit. Phys. C 65(1995)135; E. Papageorgiu, "Symmetries at ultra-high energies and searches for neutrino oscillations", LPTHE-ORSAY Preprint 93-51, Phys. Lett. B 343(1995)263.

[6] L.E. Ibáñez, Phys. Lett. B 303(1993)55. 